\title{
Episodic Memory Retrieval Benefits from a Less Modular Brain Network Organization
}

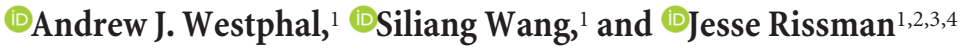 \\ ${ }^{1}$ Department of Psychology, ${ }^{2}$ Department of Psychiatry and Biobehavioral Sciences, ${ }^{3}$ Brain Research Institute, and ${ }^{4}$ Integrative Center for Learning and \\ Memory, University of California Los Angeles, Los Angeles, California 90095
}

\begin{abstract}
Most complex cognitive tasks require the coordinated interplay of multiple brain networks, but the act of retrieving an episodic memory may place especially heavy demands for communication between the frontoparietal control network (FPCN) and the default mode network (DMN), two networks that do not strongly interact with one another in many task contexts. We applied graph theoretical analysis to task-related fMRI functional connectivity data from 20 human participants and found that global brain modularity - a measure of network segregation-is markedly reduced during episodic memory retrieval relative to closely matched analogical reasoning and visuospatial perception tasks. Individual differences in modularity were correlated with memory task performance, such that lower modularity levels were associated with a lower false alarm rate. Moreover, the FPCN and DMN showed significantly elevated coupling with each other during the memory task, which correlated with the global reduction in brain modularity. Both networks also strengthened their functional connectivity with the hippocampus during the memory task. Together, these results provide a novel demonstration that reduced modularity is conducive to effective episodic retrieval, which requires close collaboration between goal-directed control processes supported by the FPCN and internally oriented self-referential processing supported by the DMN.
\end{abstract}

Key words: fMRI; functional connectivity; graph theory; memory retrieval; modularity; source memory

\section{Significance Statement}

Modularity, an index of the degree to which nodes of a complex system are organized into discrete communities, has emerged as an important construct in the characterization of brain connectivity dynamics. We provide novel evidence that the modularity of the human brain is reduced when individuals engage in episodic memory retrieval, relative to other cognitive tasks, and that this state of lower modularity is associated with improved memory performance. We propose a neural systems mechanism for this finding where the nodes of the frontoparietal control network and default mode network strengthen their interaction with one another during episodic retrieval. Such across-network communication likely facilitates effective access to internally generated representations of past event knowledge.

\section{Introduction}

Given the highly distributed nature of memory representations in the brain (Rissman and Wagner, 2012), the act of retrieving a past episode requires the coordinated engagement of a broad set of

Received June 20, 2016; revised Feb. 14, 2017; accepted Feb. 17, 2017.

Author contributions: A.J.W. and J.R. designed research; A.J.W. performed research; A.J.W. and S.W. contributed unpublished reagents/analytic tools; A.J.W. and J.R. analyzed data; A.J.W. and J.R. wrote the paper.

This work was supported by the UCLA Division of Life Sciences. A.J.W. was supported by a UCLA Neurolmaging Training Program Fellowship subaward from the National Institutes of Health (5R90DA023422-10). We thank Martin Monti for providing scripts for implementing the graph theoretical analyses, guidance on interpretation of the results, and insightful feedback on an earlier version of this manuscript. We also thank Nicco Reggente for assistance with data processing and analysis.

The authors declare no competing financial interests.

Correspondence should be addressed to either Andrew J. Westphal or Jesse Rissman, 1285 Franz Hall, Box 951563, Los Angeles, CA 90095-1563. E-mail: awestphal@ucla.edu or rissman@psych.ucla.edu.

DOI:10.1523/JNEUROSCI.2509-16.2017

Copyright $\odot 2017$ the authors $\quad 0270-6474 / 17 / 373523-09 \$ 15.00 / 0$ cortical and medial temporal lobe regions (Jeong et al., 2015). Recent neuroimaging work has highlighted the particular importance of two large-scale neural networks in facilitating the process of episodic retrieval. The frontoparietal control network (FPCN), which predominantly consists of regions along the lateral prefrontal cortex (PFC) and intraparietal sulcus (Vincent et al., 2008; Power et al., 2011), is thought to aid in the memory search process by representing one's retrieval goals and prioritizing the processing of relevant environmental cues that might help trigger the retrieval of the desired mnemonic content, as well as monitoring the details that come back to mind in the service of an impending decision or action (Donaldson et al., 2010; Spreng et al., 2010; Nyhus and Badre, 2015). The default mode network (DMN), which includes regions of the medial PFC, posterior cingulate cortex, angular gyrus, and medial temporal lobes, is thought to play a key role in the introspective processes needed to attend to and transiently represent self-generated information, such as 
memories about one's involvement in a past event (AndrewsHanna, 2012; Raichle, 2015). Indeed, most DMN regions have been implicated as components of a "core recollection network" (Rugg and Vilberg, 2013), highlighting the close correspondence between DMN function and episodic retrieval. It was initially believed that the DMN did not collaboratively interact with neural systems involved in cognitive control, as its activity is typically anticorrelated with the FPCN and other components of the "taskpositive system" during cognitive tasks (Gusnard et al., 2001; Weissman et al., 2006), as well as during the resting state (Fox et al., 2005; Fransson, 2005; but see Spreng et al., 2013, for evidence that these networks can show positive coupling during rest). However, further fMRI work has revealed that communication between the FPCN and DMN may indeed be quite important during the execution of tasks requiring controlled access to internally generated self-referential representations (Smallwood et al., 2012; Cocchi et al., 2013), such as recollecting the context of past memories (Fornito et al., 2012) or simulating future autobiographical plans (Spreng et al., 2010; Gerlach et al., 2014). Such cross talk between these two large-scale networks raises the possibility that the interplay between goal-directed control and introspective monitoring needed for episodic retrieval may push the brain into a more globally integrated state, allowing greater fluidity of information transfer. This notion is supported by recent demonstrations that functional connectivity levels throughout much of the brain are elevated during successful episodic retrieval (Schedlbauer et al., 2014; Geib et al., 2015; King et al., 2015; Westphal et al., 2016). However, no prior study has explored the relationship of global brain connectivity and networkspecific interactions during episodic retrieval.

In the present $\mathrm{fMRI}$ study, we use the graphical theoretical construct of modularity (Newman, 2006; Sporns and Betzel, 2016) to index the degree to which the brain exhibits segregation into discrete communities, or modules, during episodic memory retrieval. While a highly modularized brain network organization likely confers advantages for the efficiency of information processing (Meunier et al., 2010), complex cognitive operations require an appropriately calibrated balance between modular and integrative processing (Bertolero et al., 2015). By comparing modularity measured during an episodic memory task with that measured during the performance of two comparably demanding non-memory tasks that share identical stimulus and response characteristics, we aim to test the hypothesis that the brain should exhibit a lower level of modularity during episodic retrieval. To the extent that lower modularity is conducive to the type of internetwork communication that we believe is critical for episodic retrieval, we further predict that modularity levels should be correlated with memory performance. Finally, we will test whether the FPCN and DMN show elevated internetwork coupling with each other, as well as with the hippocampus, during episodic retrieval.

\section{Materials and Methods}

This report presents a new analysis of fMRI data previously analyzed using univariate general linear models, multivoxel pattern classification, and seed-based connectivity methods focused on rostrolateral PFC regions (Westphal et al., 2016). The present study is motivated in part by our prior result that rostrolateral PFC showed elevated functional connectivity with much of the brain during episodic memory retrieval relative to closely matched tasks of analogical reasoning and visuospatial perception. Key features of the paradigm are described below.

Participants. Twenty healthy adult participants (10 female; mean age $=21.1$ years) from the University of California, Los Angeles (UCLA) and the surrounding community participated in this study and had suf- ficient data for analysis purposes. Written informed consent was obtained in accordance with procedures approved by the UCLA Institutional Review Board, and participants received monetary compensation for their time. All were fluent English speakers with normal or corrected-to-normal vision and no history of drug or alcohol dependence, mental illness, or contraindications to MRI.

Procedure. The experiment required participation in two sessions conducted on consecutive days. On the first day, participants performed a memory-encoding task, where they were presented with a series of 80 words, each preceded by a mental imagery context cue indicating whether they should mentally visualize themselves ("Self") or another person ("Other") interacting with the referent of the ensuing word. Participants were informed that they would later be tested on their memory for both the word and the associated imagery context. The participants then performed a computerized task where they gained proficiency in understanding the button response options for the Memory, Reasoning, and Perception tasks that they would be performing in the scanner the next day. Last, they performed a practice run of the three cognitive tasks.

On the second day, fMRI data were acquired as participants performed the three cognitive tasks, which were counterbalanced such that two blocks of the same task were never consecutively presented, and all three tasks had to be presented in each set of three blocks. The total session consisted of eight scanning runs, which each included nine $52 \mathrm{~s}$ blocks, with three blocks of each task. Blocks consisted of the presentation of a task-set cue for $6 \mathrm{~s}$, indicating which task was to be performed, followed by a $2 \mathrm{~s}$ fixation cross, and then four trials of the specified task. Each trial consisted of the presentation of a four-word stimulus array for $8 \mathrm{~s}$, during which time participants indicated their response, followed by a $2 \mathrm{~s}$ fixation cross. An extra $4 \mathrm{~s}$ of fixation was included the end of each four-trial task block. A schematic of the three-task paradigm is presented in Figure 1a. The experiment included 288 total trials, with 96 trials of each task.

The Memory, Reasoning, and Perception tasks all used four-word stimulus arrays to equate bottom-up input to the visual system and only differed in the cognitive processes required to evaluate the stimuli and arrive at an appropriate decision. The Memory task (cued by an " $M$ ") required participants to scan the four-word array and identify whether one of the words had been encountered during the memory-encoding task on the previous day (75\% of trials contained a studied word; $25 \%$ contained all novels words). Participants were encouraged to specify the associated encoding context, if they remembered it. The response options were as follows: (1) Remember One of the Words from SELF Context; (2) Remember One of the Words from OTHER Context; (3) Recognize One of the Words, But Don't Recall Source; (4) All Words are Novel. The Reasoning task (cued by an "R") required participants to assess whether the top word pair and bottom word pair formed an analogical relationship or, if not, to indicate how many semantic relationships were present ( $50 \%$ of trials contained a valid analogical relationship; $16.7 \%$ contained two non-analogous semantic relationships; $16.7 \%$ contained one semantic relationship; $16.7 \%$ contained no semantic relationships). The response options were as follows: (1) Valid Analogical Relationship; (2) Two Valid Semantic Relationships; (3) One Valid Semantic Relationship; (4) No Semantic Relationships. The Perception task (cued with a "P") required participants to judge which word, printed in lower-case Geneva font, contained the greatest number of straight lines within its letters. The words in each array were chosen such that one always had $\geq 2$ more straight lines than any of the others. The response options were as follows: (1) Top Left Word Has the Most Straight Lines; (2) Bottom Left Word Has the Most Straight Lines; (3) Bottom Right Word Has the Most Straight Lines; (4) Top Right Word Has the Most Straight Lines.

MRI data acquisition and fMRI preprocessing. Whole-brain MRI was administered on a 3.0 Tesla Siemens TIM Trio scanner at the UCLA Staglin Center for Cognitive Neuroscience. Functional images were acquired using a $\mathrm{T} 2^{*}$-weighted echoplanar imaging sequence $(\mathrm{TR}=2.0 \mathrm{~s}$; $\mathrm{TE}=30 \mathrm{~ms}$; flip angle $=75^{\circ} ; \mathrm{FOV}=19.2 \mathrm{~cm}$; voxel resolution $=3.0 \times$ $3.0 \times 3.7 \mathrm{~mm} ; 33$ interleaved axial slices). The first three volumes of each 239-volume run were discarded to ensure T1 stabilization. To facilitate the spatial registration of the functional images, we acquired a highresolution $\left(1 \mathrm{~mm}^{3}\right)$ T1-weighted anatomical scan and a coplanar T2- 
a
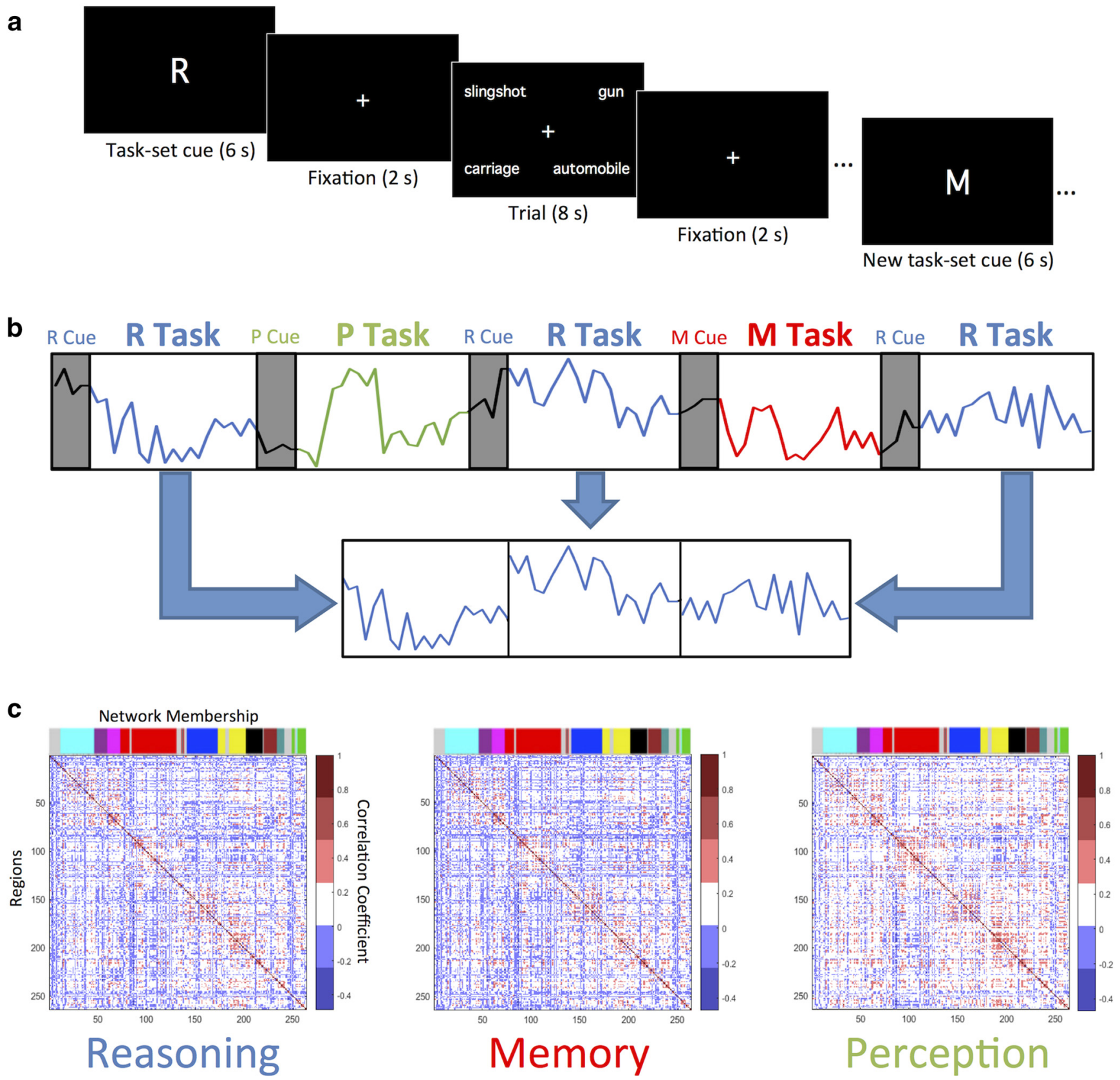

Figure 1. Schematic representation of cognitive task paradigm, procedure for creating task-specific timecourses and mean task-specific correlation matrices. $\boldsymbol{a}$, Task blocks begin with the presentation of a task-set cue (M, R, or P) specifying which task (Memory, Reasoning, or Perception) should be performed during the following four trials. Each trial consists of a four-word array, and participants have $8 \mathrm{~s}$ to respond with a button press. Trials were separated by brief periods of resting fixation. After the end of each four-trial task block, a new task-set cue appears, followed by four trials of the new task. $\boldsymbol{b}$, Task-specific timecourses were created by concatenating task blocks (twenty-four $38 \mathrm{~s}$ blocks per task) for each node, excluding cue-period activity. In this schematic example, three Reasoning task blocks were concatenated. c, Mean correlation matrices illustrate the pairwise correlations among all 264 nodes for each task, averaged across participants. The colored bands along the top of each plot indicate the network membership of the 264 nodes, as specified by Power et al. (2011).

weighted anatomical scan. Additionally, to aid in unwarping of anterior regions of the frontal and temporal lobes, we collected a field map scan representing magnetic field inhomogeneities.

Image preprocessing was performed with SPM8 (RRID:SCR_007037; http://www.fil.ion.ucl.ac.uk/spm/software/spm8/) and custom Matlab (RRID:SCR_001622) code. Preprocessing included slice-time correction, motion correction, unwarping, coregistration, anatomical segmentation, spatial normalization to the MNI template, spatial smoothing $(6 \mathrm{~mm}$ FWHM), and high-pass filtering (cutoff period $=236 \mathrm{~s})$. The head-motion estimates were then used as regressors and the residuals were saved for further analysis. Last, each voxel's timecourse was demeaned for each scanning run.
Task-specific functional connectivity estimation. After the functional data were preprocessed, task-specific timecourses were extracted for each task and each subject from a set of 264 nodes identified in a meta-analysis and resting-state parcellation study by Power and colleagues (2011). To create task-specific timecourses, we excised 38 s (19 TRs) of data from each task block (beginning $10 \mathrm{~s}$ after cue onset and ending $2 \mathrm{~s}$ after the offset of the fourth trial) and concatenated these segments across the 24 blocks of each task condition, resulting in 456 TRs of data for each task (Fig. 1b). We then averaged the timecourses within all voxels of each node, and then correlated these mean timecourses across all pairs of nodes to yield a $264 \times 264$ correlation matrix for each task and each subject (Fig. 1c). Note that because all three tasks had identical stimulus 
characteristics and event timing parameters, functional connectivity differences between tasks are unlikely to be due to differences in bottom-up perceptual stimulation. However, to further protect against the possibility of our graph theoretic metrics for each task being systematically influenced by the properties of the generic task model, we regressed out the timecourse of all task events and performed the functional connectivity analysis on the residuals. This procedure for obtaining estimates of task-dependent connectivity independent of signal fluctuations driven by the onset/offset of stimulus events is sometimes referred to as "background connectivity" analysis (Al-Aidroos et al., 2012; Córdova et al., 2016). The task timecourse model was specified by convolving a boxcar model for all task events (with durations of $6 \mathrm{~s}$ for cues and $8 \mathrm{~s}$ for word arrays) with SPM8's canonical hemodynamic response function. Although we report the findings after the taskrelated timecourse was regressed from the data, we note that all results below are replicated without this analysis step. To improve normality and facilitate statistical testing, each subject's matrix of Pearson's $r$ values was Fisher-transformed before further analysis.

Graph theoretic analysis. We used the Brain Connectivity Toolbox (RRID:SCR_004841; https://sites.google.com/site/bctnet/; Rubinov and Sporns, 2010) to estimate several graph theoretic metrics of interest from each correlation matrix. The graph theory metrics were measured at a range of sparsity levels for each task, ranging from a lower bound of the top $4 \%$ of correlation strengths to an upper bound of the top $20 \%$ of correlation strengths, in 2\% intervals (including 6, 8, 10, 12, 14, 16, and $18 \%)$. The upper bound of $20 \%$ was selected as this was the densest graph where each task had small-worldness levels significantly $>1$ for each task, with small-worldness defined as normalized clustering divided by normalized path length. The lower bound of $4 \%$ was chosen due to this being the sparsest graph where small-worldness was computable for each subject and task. Clustering and path length were normalized by dividing the obtained metric with the average metric obtained in 10 randomly initialized networks. All graph theory metrics were aggregated across the different sparsity thresholds by calculating the area under the curve (AUC) for each metric (Bassett and Lynall, 2014).

Modularity (Q), which reflects how effectively community detection algorithms (Fortunato, 2010) can partition the correlation matrices into communities of nodes that demonstrate primarily within-module connectivity, was calculated for all three tasks and all subjects using the Louvain algorithm (Blondel et al., 2008). In addition to comparing modularity across tasks, we tested our hypothesis that individual differences in modularity scores might be related to Memory task performance. To this end, we correlated modularity with three behavioral performance metrics [mnemonic discriminability $\left(d^{\prime}\right)$, hit rate, and false alarm rate]. The $d$ ' measure is calculated by subtracting the false alarm rate from the hit rate, with both measures normalized using the inverse of the cumulative standardized normal distribution. The hit rate was defined as the proportion of trials containing a studied word for which the participant correctly indicated that a studied word was present (regardless of source accuracy). The false alarm rate was defined as the proportion of trials not containing a studied word for which the participant falsely reported recognition (with or without source details).

We directly calculated the mean correlation strength between the nodes of the FPCN and DMN to assess the degree to which the interaction between these two large-scale networks can be linked to global modularity levels. The average transformed correlation among all $25 \mathrm{FPCN}$ nodes and $58 \mathrm{DMN}$ nodes was computed for each task. Given the critical role of the hippocampus in memory, we also examined the mean strength of FPCN and DMN coupling with the hippocampus, which was treated as a network consisting of two nodes [because the 264-node parcellation used in our analyses did not include hippocampal nodes, we defined nodes in the left and right hippocampus using the Harvard-Oxford atlas (RRID:SCR_001476) with a probability threshold of 50\%].

\section{Results}

\section{Behavioral results}

The distribution of responses across trial types within each task condition, as well as the associated response times, has been previously reported in full (Westphal et al., 2016); key summary statistics are reproduced here. Overall accuracy for the Memory task (i.e., proportion of trials on which participants correctly indicated whether the word array contained a studied word) was $75.1 \%$ (mean hit rate $=0.77$; mean false alarm rate $=0.30$; mean $d^{\prime}=1.37$ ). Although participants only reported a memory for the source context on $60.0 \%$ of trials containing an old word, when they did so they were highly accurate, selecting the correct source $82.3 \%$ of the time. Overall accuracy for the Reasoning task (i.e., proportion of trials on which participants correctly indicated whether the word array contained a valid analogy, or two, one, or no semantic relationships) was $76.4 \%$. Overall accuracy for the Perception task (i.e., proportion of trials on which participants correctly indicated which word contained the greatest number of straight lines) was $66.9 \%$. Mean response times ( \pm SE) across all task trials were as follows: Memory $=5.04 \mathrm{~s}(0.15)$; Reasoning $=$ $4.72 \mathrm{~s}$ (0.10); Perception $=5.03 \mathrm{~s}(0.19)$.

\section{Task-dependent changes in global connectivity}

Modularity is a global graph theoretic measure that indexes how well functional connectivity of the brain can be partitioned into distinct communities (groups of nodes that interact more strongly with each other than with the rest of the network). A high modularity value indicates that distinct communities, or modules, interact minimally with one another, whereas a lower modularity value suggests stronger intermodular interactions. Our graph analysis results found that brain connectivity during the Memory task exhibited significantly lower modularity than during both the Reasoning and Perception tasks (Fig. 2a); paired $t$ tests confirmed this result at every sparsity level [all $p$ 's $<0.05$ after Benjamini-Hochberg false discovery rate correction for 18 tests, with the exception of one test (Reasoning $>$ Memory at $4 \%$ sparsity) that only showed trend level significance $(p=0.068)]$. Modularity estimates were aggregated across the nine sparsity thresholds using an AUC metric and compared across tasks (Fig. 2b). A repeated-measures ANOVA demonstrated a significant effect of task using the modularity AUC measure $\left(F_{(2,18)}=5.64, p=\right.$ 0.013). Post hoc tests showed that the Memory task exhibited reduced modularity compared with the Reasoning $(p=0.008)$ and Perception $(p=0.003)$ tasks, with the latter two conditions showing no difference from each other $(p=0.844) ; \alpha_{\text {crit-Bonferroni }}=0.017$. Although mean modularity levels showed task-dependent changes, we note that individual differences in modularity were strongly correlated across tasks $r_{\text {(Reasoning, Memory) }}=0.821, p<0.001$; $r_{\text {(Reasoning, Perception) }}=0.904, p<0.001 ; r_{\text {(Memory, Perception) }}=0.841$, $p<0.001$, indicating that overall brain modularity may be a trait characteristic whose magnitude can systematically increase or decrease across cognitive task states.

Given our finding that modularity was significantly reduced during the Memory task, we next examined whether modularity levels were related to participants' behavioral performance on this task. Memory modularity scores were negatively correlated with memory $d$ ' scores, although this trend narrowly failed to reach significance $(r=-0.426, p=0.061)$. When $d$ ' was dissected into its hit rate and false alarm rate components, there was no correlation observed between Memory modularity and hit rates $(r=0.120, p=0.616)$, but false alarm rates showed a significant positive correlation with modularity $(r=0.551, p=$ $0.012 ; \alpha_{\text {crit-Bonferroni }}=0.017 ; 95 \%$ confidence interval (bootstrapped): $[0.145,0.794])$. This brain-behavior relationship, shown in Figure $2 c$, demonstrates that individuals with higher modularity tend to make more false alarm errors on the Memory task (i.e., reporting recognition or recollection of a word on trials where no studied words were present in the array). 

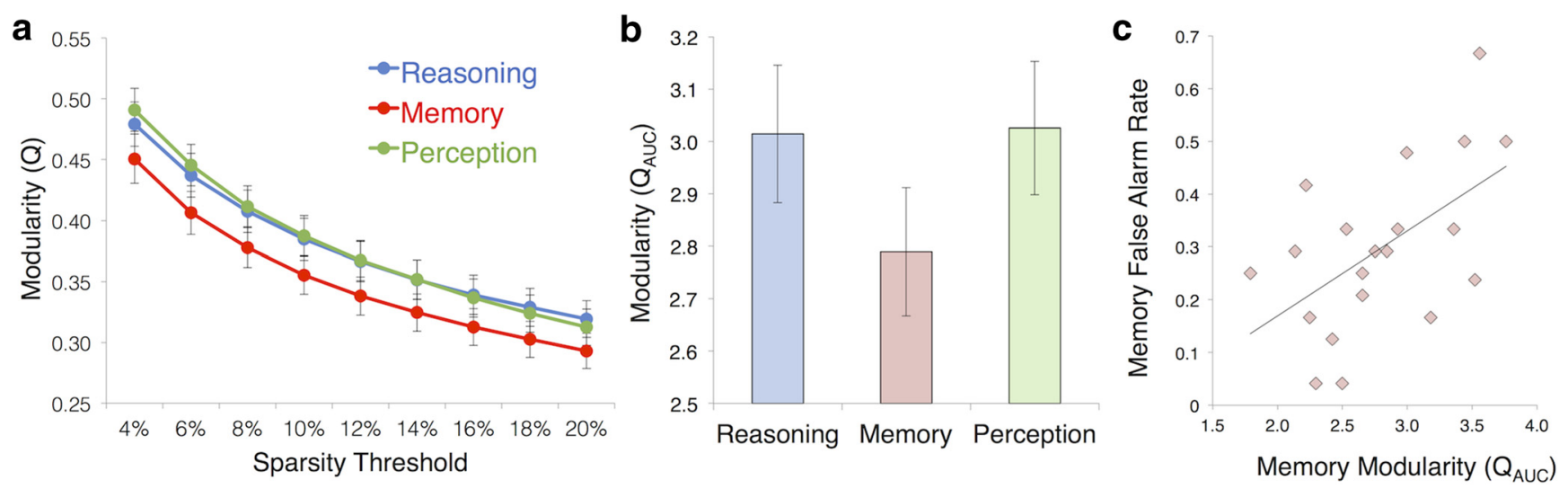

Figure 2. Modularity is reduced during Memory and related to task performance. $\boldsymbol{a}$, Modularity levels for each task and threshold demonstrate that modularity during Memory is significantly and consistently reduced compared with Reasoning and Perception at each sparsity threshold. $\boldsymbol{b}$, Comparison of mean modularity across tasks, revealing a significant reduction during Memory relative to Reasoning and Perception. $c$, Scatter plot showing the correlation between brain modularity and false alarm rates during the Memory task $(r=0.551, p=0.012)$. Error bars indicate SEM.
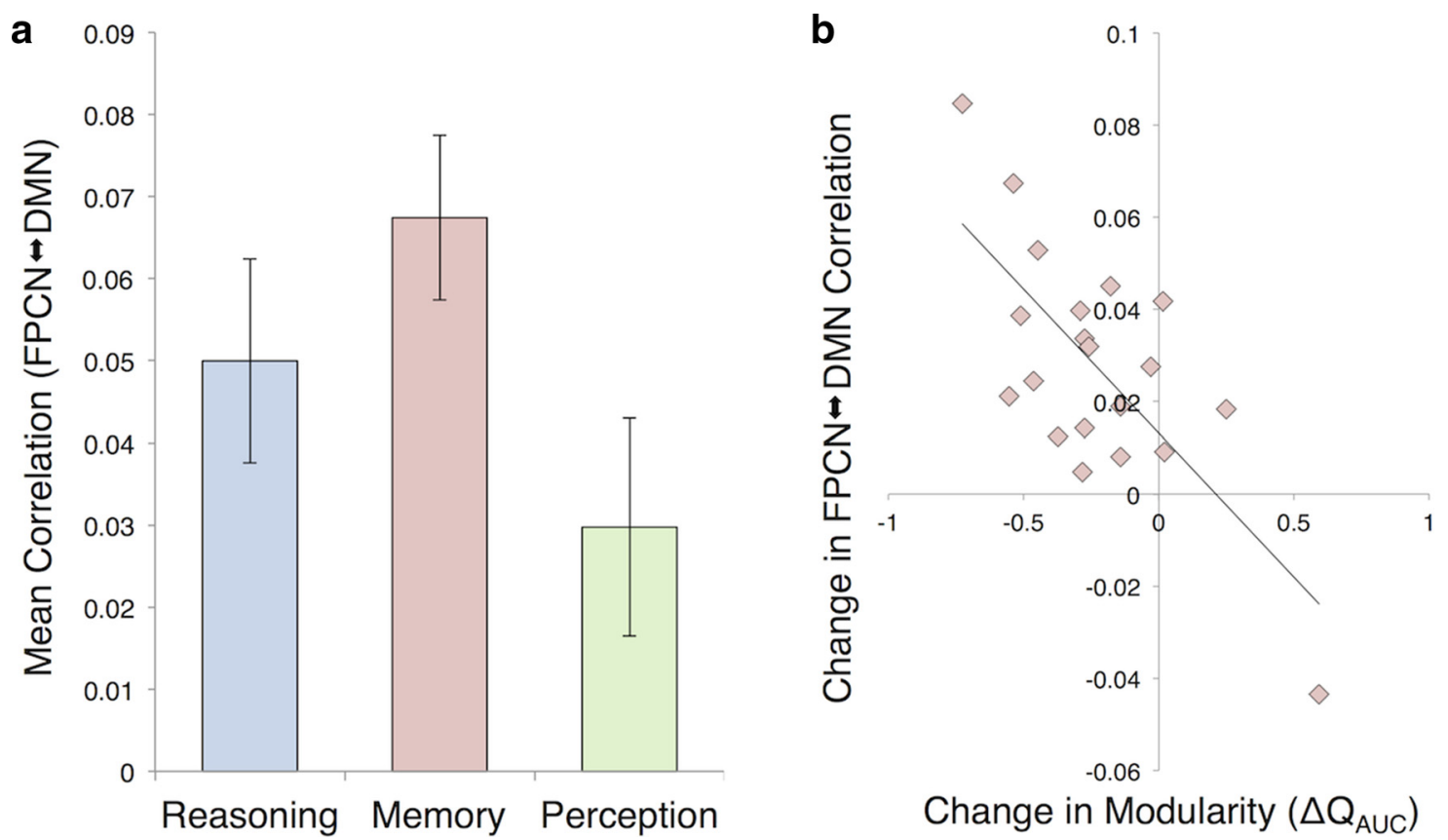

Figure 3. The FPCN and DMN strengthen their coupling with each other during Memory, and the magnitude of this internetwork strengthening correlates with decreased modularity during Memory. $\boldsymbol{a}$, The FPCN and DMN show higher coupling during the Memory task than during the Reasoning and Perception tasks. $\boldsymbol{b}$, The scatter plot illustrates that those individuals whose brains showed larger increases in across-network connectivity between the FPCN and DMN during Memory (relative to the average level observed during Reasoning and Perception) showed a correlated reduction in modularity during Memory (relative to the average level observed during Reasoning and Perception; $r=-0.718, p<0.001$ ). Error bars indicate SEM.

\section{Across-network correlations}

We next aimed to determine whether the connectivity strength between our networks of interest, the FPCN and DMN, would be elevated in the Memory task. We assessed this by computing the average connectivity strength (Fisher-transformed correlation coefficient) between all pairs of FPCN and DMN nodes, excluding all intranetwork connections, during each task condition (Fig. 3a). A repeated-measures ANOVA revealed a significant effect of task on FPCN-DMN connectivity $\left(F_{(2,18)}=21.76, p<\right.$ 0.001 ); all post hoc paired comparisons were significant (Memory $>$ Reasoning, $p=0.008$; Memory $>$ Perception, $p<0.001$; Reasoning $>$ Perception, $\left.p<0.001 ; \alpha_{\text {crit-Bonferroni }}=0.017\right)$. To determine whether this effect was specific to connectivity between the FPCN and DMN, we examined the connectivity properties of two additional large-scale networks: the cinguloopercular network (CON), implicated in the maintenance of sta- ble cognitive task sets (Dosenbach et al., 2008), and the dorsal attention network (DAN), implicated in the top-down control of visuospatial attention (Corbetta and Shulman, 2002). The CON did not show any task-dependent changes in its connectivity strength with the other networks (CON-FPCN, $F_{(2,18)}=$ $2.22, p=0.138 ; \mathrm{CON}-\mathrm{DMN}, F_{(2,18)}=1.79, p=0.195$; CON-DAN, $\left.F_{(2,18)}=0.40, p=0.678\right)$. The DAN did show task-dependent changes in connectivity with the FPCN $\left(_{(2,18)}=13.10, p<0.001\right)$ and $\operatorname{DMN}\left(F_{(2,18)}=23.63, p<\right.$ $0.001)$, but these effects were driven by its connectivity levels during the Perception task. Specifically, the DAN showed increased coupling with the FPCN during Perception relative to both Memory $(p<0.001)$ and Reasoning $(p=0.003)$, whereas the DAN showed decreased coupling with the DMN during Perception relative to both Memory $(p<0.001)$ and Reasoning $(p<0.001)$; in neither case did its mean connec- 

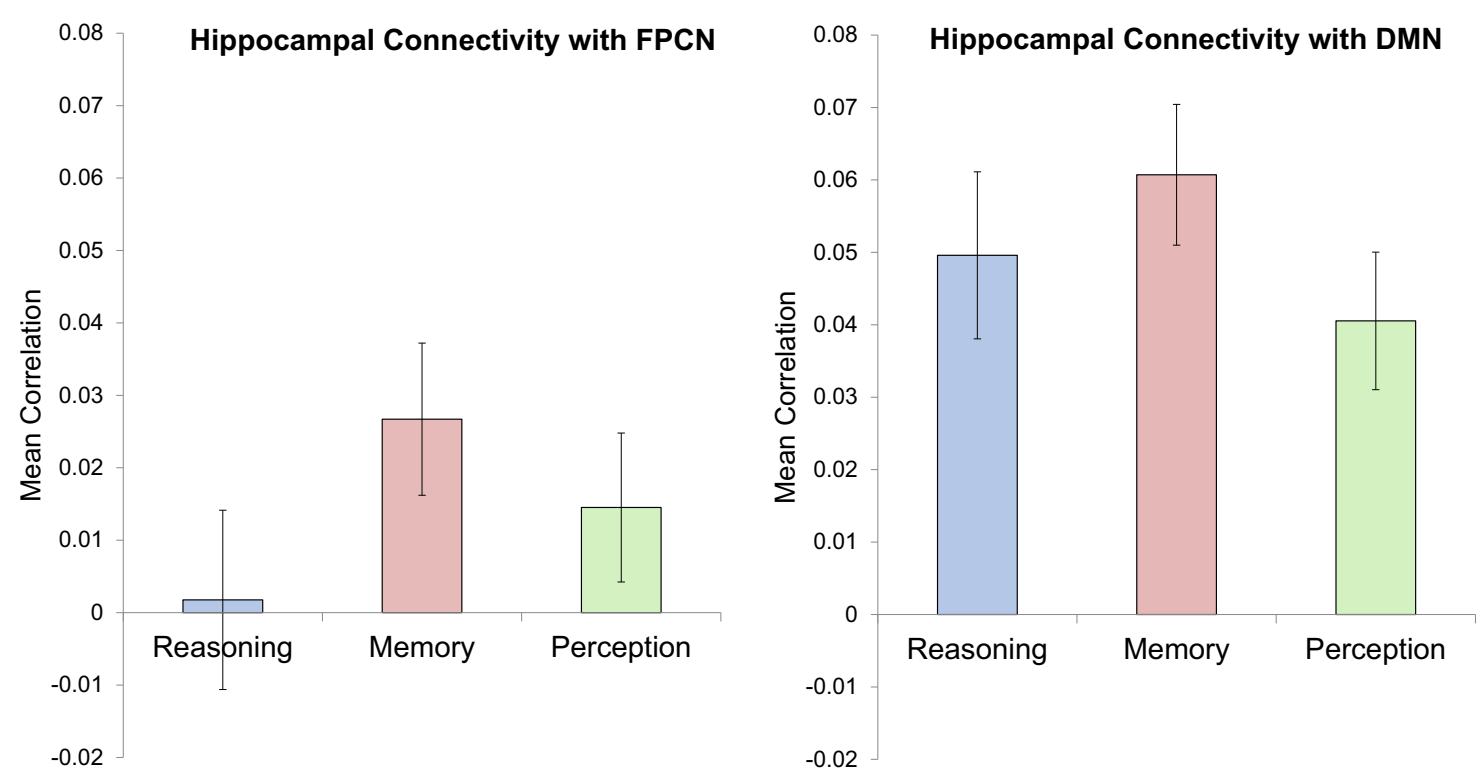

Figure 4. Hippocampal connectivity with the FPCN and DMN is strengthened during Memory. The main effect of task demonstrates that the hippocampus exhibits increased coupling with the FPCN and DMN in the Memory task compared with the Reasoning and Perception tasks, consistent with a potential hub-like role in memory retrieval (Geib et al., 2015). Additionally, the main effect of network shows that the hippocampus exhibits preferential coupling with the DMN relative to its connectivity strength with the FPCN, consistent with resting-state connectivity studies (Vincent et al., 2006). Error bars indicate SEM.

tivity level during Memory numerically exceed nor statistically differ from that of Reasoning (all p's >0.1).

Given our finding of heightened internetwork connectivity between the FPCN and DMN during the Memory task, we were interested in whether this effect was related to our earlier finding of decreased global brain modularity during Memory. To examine whether these effects were related, we correlated individual differences in the degree to which FPCN-DMN connectivity was increased during Memory (relative to its mean value during Reasoning and Perception) with the degree to which modularity was decreased during Memory (relative to its mean value during Reasoning and Perception). The correlation between these change scores was significantly negative $(r=-0.718$, $p<0.001 ; 95 \%$ confidence interval (bootstrapped): [ -0.893 , $-0.153]$ Fig. $3 b)$. Inspection of the scatter plot led to the identification of an outlier in the lower right quadrant; however, the correlation remained significant when this data point was removed $(r=-0.526, p=0.021)$.

\section{FPCN and DMN coupling with hippocampus}

While our core hypotheses have focused on connectivity effects within large-scale networks rather than individual brain regions, we were interested in examining the degree to which these networks modulated the strength of their coupling with the hippocampus as a function of participants' cognitive task set. Because the Power et al. (2011) 264-node parcellation used in our analyses did not include hippocampal nodes, we explored hippocampal connectivity by defining ROIs in the left and right hippocampus using the Harvard-Oxford atlas. We then computed the mean pairwise connectivity between each hippocampal node and all FPCN and DMN nodes and submitted the results to a network $\times$ task repeated-measures ANOVA (Fig. 4). Consistent with prior resting-state connectivity results showing that the hippocampi are closely linked with the DMN (Vincent et al., 2006; Andrews-Hanna et al., 2010), we found a main effect of network $\left(F_{(1,19)}=19.91, p<0.001\right)$, whereby hippocampal connectivity was significantly greater with DMN nodes than with FPCN nodes. We also found a main effect of task $\left(F_{(2,18)}=7.66, p=\right.$ $0.004)$, driven by elevated activity during the Memory task relative to its mean level during Reasoning and Perception. And finally, we found a significant network $\times$ task interaction $\left(F_{(2,18)}=\right.$ $4.12, p=0.034)$, such that the Memory advantage in hippocampal connectivity with the FPCN was more pronounced relative to Reasoning $(p=0.011)$ than relative to Perception $(p=0.057)$, whereas the Memory advantage in hippocampal connectivity with the DMN was more pronounced relative to Perception $(p<0.001)$ than relative to Reasoning $(p=0.059)$. We also examined the specificity of the FPCN and DMN interactions with the hippocampus by comparing them to the effects obtained when running the same analysis using the CON and DAN. Repeated-measures ANOVAs assessing task-related connectivity were not significant for the connectivity between the hippocampus and the $\operatorname{CON}\left(F_{(2,18)}=2.03, p=0.161\right)$ or $\operatorname{DAN}\left(F_{(2,18)}=0.92, p=0.418\right)$.

\section{Discussion}

Our study used graph theoretic modeling to evaluate the wholebrain fMRI functional connectivity properties associated with episodic memory retrieval. Importantly, our unique experimental protocol allowed us to compare these properties to those measured when the same participants performed two non-episodic tasks with identical stimulus presentation characteristics and highly comparable response demands. We found that global brain modularity, an index of network segregation, was markedly reduced during episodic retrieval relative to the Reasoning and Perception tasks and was associated with improved memory performance. Further analysis indicated that this reduction in modularity was strongly associated with increased connectivity between the FPCN and DMN networks.

Although several recent fMRI studies have examined how brain modularity is affected by changes in memory-related task demands, such as comparisons of load levels of an $\mathrm{N}$-back working memory task (Stanley et al., 2014; Vatansever et al., 2015; Liang et al., 2016), our study is the first to directly compare how modularity differs between memory and other complex cognitive 
tasks. Our finding that modularity was consistently lower during episodic retrieval than during analogical reasoning or visuospatial processing suggests that the act of retrieving an episodic memory requires a highly integrated brain state where the nodes of seemingly distinct modules are more likely to interact with nodes outside their own designated modules. At first glance it might seem surprising that a complex cognitive task like analogical reasoning did not elicit a comparably integrated brain state, given its demands for deriving and comparing abstract semantic relationships. However, the present findings are consistent with a prior seed-based connectivity analysis of these data, which also indicated that analogical reasoning engaged a more anatomically circumscribed network than that engaged during episodic retrieval (Westphal et al., 2016). Specifically, when directly comparing functional connectivity during reasoning and memory, Westphal et al. (2016) found that left rostrolateral PFC, a region commonly used as a seed for identifying the FPCN (Vincent et al., 2008), showed significantly heightened connectivity with only two brain regions during reasoning: a nearby prefrontal region (left BA 45) putatively involved in semantic processing and a region of the superior parietal lobule. In contrast, dozens of large clusters throughout the FPCN and DMN showed elevated connectivity with the left rostrolateral PFC during memory relative to reasoning. Thus, while it is unquestionable that analogical reasoning requires the interactivity of multiple brain systems (Knowlton et al., 2012), our data suggest that the demands for across-network communication are even greater during episodic retrieval. It is possible that many of the challenging computations needed to solve analogies can be accomplished by neural circuits within the PFC, and thus globally enhanced brain connectivity need not necessarily be a hallmark of analogical reasoning.

The enhancement of large-scale network connectivity associated with episodic retrieval was clearly evident in our follow-up analysis examining the connectivity between our two principal networks of interest. Not only did the nodes of the FPCN and DMN strengthen their connectivity with each other during memory, but the magnitude of this increased internetwork coupling correlated with the magnitude of the modularity reduction effect. Although increased FPCN-DMN coupling is unlikely to be the sole factor underlying the global modularity reduction, the strong correlation between these respective metrics suggests that it may be a major contributor to this phenomenon. The heightened interactivity between the FPCN and DMN during episodic retrieval is consistent with prior fMRI findings from Spreng and colleagues (2010), who used a partial least-squares analysis approach to reveal greater coactivation of these two networks during an autobiographical planning task than during similarly structured tasks involving visuospatial planning or counting. Our finding is also consistent with the results of Fornito and colleagues (2012), who compared connectivity during episodic retrieval to connectivity during a pseudo-resting-state condition. They found that while the DMN interacted minimally, if not antagonistically, with FPCN regions during rest, its nodes became more tightly coupled with FPCN nodes during episodic retrieval.

While such cooperative interactions between the FPCN and DMN appear to be a hallmark of episodic retrieval, the interplay between these networks may be more broadly conceptualized as facilitating the controlled generation and maintenance of an internally oriented train of thought (Smallwood et al., 2012). According to this view, the FPCN may function as something akin to a "global workspace" (Dehaene et al., 1998) capable of augmenting or suppressing inputs from other networks in accordance with one's current goals and, in so doing, specifying which representations will dominate the contents of conscious processing. When processing is focused on information in the sensory environment, connectivity between the FPCN and the externally oriented dorsal attention network will dominate. However, when the demands of a task require the monitoring of self-generated information (i.e., information that cannot be readily extracted from the immediate sensory environment), the FPCN will strengthen its coupling with the DMN.

In addition to our finding of strengthened FPCN-DMN coupling, we found that both networks showed increased connectivity with the hippocampus during episodic retrieval. Although the hippocampus is often considered a component region of the DMN (Vincent et al., 2006; Andrews-Hanna et al., 2010; Raichle, 2015), hippocampal connectivity with DMN regions has been found to be task-dependent, with high coupling observed during episodic retrieval and resting-state conditions, but minimal coupling detected during encoding (Huijbers et al., 2011). Our analyses showed that while the hippocampus interacted more strongly with the DMN during all three cognitive tasks, its interactions with both the DMN and FPCN were significantly stronger during the Memory task. This finding is generally consistent with recent graph-theoretical demonstrations that the hippocampus fulfills a hub-like role, flexibly interacting with nodes of multiple cortical networks to facilitate successful episodic retrieval (Schedlbauer et al., 2014; Geib et al., 2015).

Another noteworthy result from the present investigation was the correlation between participants' modularity levels and their behavioral performance on the Memory task. Specifically, participants with lower modularity tended to make fewer false alarms on the Memory task (i.e., they were less likely to report the presence of a studied word on trials featuring word arrays with all novel words). It is unclear why this brain-behavior relationship was most strongly manifested in the false alarm rate data, as we would have also predicted an effect on participants' ability to correctly retrieve memories for trials containing old items (i.e., increased hit rate). Future work with larger samples may be need to determine whether reduced modularity confers a generalized advantage for retrieval task performance (we note that we did find a nearly significant negative correlation between modularity and overall memory $d^{\prime}$ ), or whether the advantage is indeed selective to false alarms, perhaps by bolstering the mnemonic control processes necessary to correctly reject the novel foils. That said, our finding that lower modularity was beneficial for memory performance is largely consistent with several recent reports. Meunier and colleagues (2014) examined functional connectivity across a smaller network of 36 regions during an olfactory recognition memory task, and they too observed a negative correlation between modularity and performance. Another recent study compared modularity across load levels in an $n$-back workingmemory task and found that increased memory load was associated with lower modularity, which in turn was associated with faster reaction times for correct responses (Vatansever et al., 2015). An N-back working memory study by Stanley and colleagues (2014) found no overall change in modularity as a function of load, yet did find that subjects who performed best on the more challenging two-back condition tended to show reduced modularity during that condition. Intriguingly, several other studies that have measured participants' modularity levels during resting-state fMRI scans have found the opposite relationship between modularity and performance on memory tasks (performed outside the scanner). Two such studies found that higher modularity was associated with better performance on visuospa- 
tial working memory tasks (Stevens et al., 2012; Alavash et al., 2015), and one study, which used a graph theoretical metric of network segregation, found that higher segregation of association networks predicted improved performance in verbal episodic memory (Chan et al., 2014).

Further work will be needed to evaluate whether this apparent divergence in the relationship between task-related and resting-state modularity levels and memory performance is a meaningful phenomenon. Our results suggest that, at least when measured during cognitive task performance, modularity levels may reflect a trait characteristic, in that participants' modularity scores were highly correlated across the three tasks. In this sense, the reduction in modularity associated with episodic retrieval represents a transient modulation of one's network properties from their "baseline" state. However, it remains unclear how the task-related baseline (putatively indexed by the Reasoning and Perception tasks in our experiment) may differ from a resting baseline state, or from other cognitive task states. Although our three tasks were carefully structured so as to share a common set of stimulus and response characteristics, other cognitive tasks may be difficult to constrain in this way, making comparisons challenging. Still, we believe that our results provide a valuable foundation for future research, showcasing the potential of a graph theoretical analysis approach to provide valuable insights into the brain network properties that underlie complex cognition.

\section{References}

Al-Aidroos N, Said CP, Turk-Browne NB (2012) Top-down attention switches coupling between low-level and high-level areas of human visual cortex. Proc Natl Acad Sci U S A 109:14675-14680. CrossRef Medline

Alavash M, Doebler P, Holling H, Thiel CM, Gießing C (2015) Is functional integration of resting state brain networks an unspecific biomarker for working memory performance? Neuroimage 108:182-193. CrossRef Medline

Andrews-Hanna JR (2012) The brain's default network and its adaptive role in internal mentation. Neuroscientist 18:251-270. CrossRef Medline

Andrews-Hanna JR, Reidler JS, Sepulcre J, Poulin R, Buckner RL (2010) Functional-anatomic fractionation of the brain's default network. Neuron 65:550-562. CrossRef Medline

Bassett DS, Lynall M-E (2014) Network methods to characterize brain structure and function. In: The cognitive neurosciences, fourth edition (Gazzaniga MS, Mangun GR, eds), pp 935-948. Cambridge,MA: MIT.

Bertolero MA, Yeo BT, D’Esposito M (2015) The modular and integrative functional architecture of the human brain. Proc Natl Acad Sci U S A 112:E6798-E6807. CrossRef Medline

Blondel VD, Guillaume J, Lambiotte R, Lefebvre E (2008) Fast unfolding of communities in large networks. J Stat Mech-Theory E 2008:P10008. CrossRef

Chan MY, Park DC, Savalia NK, Petersen SE, Wig GS (2014) Decreased segregation of brain systems across the healthy adult lifespan. Proc Natl Acad Sci U S A 111:E4997-E5006. CrossRef Medline

Cocchi L, Zalesky A, Fornito A, Mattingley JB (2013) Dynamic cooperation and competition between brain systems during cognitive control. Trends Cogn Sci 17:493-501. CrossRef Medline

Corbetta M, Shulman GL (2002) Control of goal-directed and stimulusdriven attention in the brain. Nat Rev Neurosci 3:201-215. Medline

Córdova NI, Tompary A, Turk-Browne NB (2016) Attentional modulation of background connectivity between ventral visual cortex and the medial temporal lobe. Neurobiol Learn Mem 134:115-122. CrossRef Medline

Dehaene S, Kerszberg M, Changeux JP (1998) A neuronal model of a global workspace in effortful cognitive tasks. Proc Natl Acad Sci U S A 95: 14529-14534. CrossRef Medline

Donaldson DI, Wheeler ME, Petersen SE (2010) Remember the source: dissociating frontal and parietal contributions to episodic memory. J Cogn Neurosci 22:377-391. CrossRef Medline

Dosenbach NU, Fair DA, Cohen AL, Schlaggar BL, Petersen SE (2008) A dual-networks architecture of top-down control. Trends Cogn Sci 12:99105. CrossRef Medline

Fornito A, Harrison BJ, Zalesky A, Simons JS (2012) Competitive and cooperative dynamics of large-scale brain functional networks supporting recollection. Proc Natl Acad Sci U S A 109:12788-12793. CrossRef Medline

Fortunato S (2010) Community detection in graphs. Phys Rep 486:75-174. CrossRef

Fox MD, Snyder AZ, Vincent JL, Corbetta M, Van Essen DC, Raichle ME (2005) The human brain is intrinsically organized into dynamic, anticorrelated functional networks. Proc Natl Acad Sci U S A 102:9673-9678. CrossRef Medline

Fransson P (2005) Spontaneous low-frequency BOLD signal fluctuations: an fMRI investigation of the resting-state default mode of brain function hypothesis. Hum Brain Mapp 26:15-29. CrossRef Medline

Geib BR, Stanley ML, Wing EA, Laurienti PJ, Cabeza R (2015) Hippocampal contributions to the large-scale episodic memory network predict vivid visual memories. Cereb Cortex pii:bhv272. CrossRef Medline

Gerlach KD, Spreng RN, Madore KP, Schacter DL (2014) Future planning: default network activity couples with frontoparietal control network and reward-processing regions during process and outcome simulations. Soc Cogn Affect Neurosci 9:1942-1951. CrossRef Medline

Gusnard DA, Raichle ME, Raichle ME (2001) Searching for a baseline: functional imaging and the resting human brain. Nat Rev Neurosci 2:685694. CrossRef Medline

Huijbers W, Pennartz CM, Cabeza R, Daselaar SM (2011) The hippocampus is coupled with the default network during memory retrieval but not during memory encoding. PLoS One 6:e17463. CrossRef Medline

Jeong W, Chung CK, Kim JS (2015) Episodic memory in aspects of largescale brain networks. Front Hum Neurosci 9:454. CrossRef Medline

King DR, de Chastelaine M, Elward RL, Wang TH, Rugg MD (2015) Recollection-related increases in functional connectivity predict individual differences in memory accuracy. J Neurosci 35:1763-1772. CrossRef Medline

Knowlton BJ, Morrison RG, Hummel JE, Holyoak KJ (2012) A neurocomputational system for relational reasoning. Trends Cogn Sci 16:373-381. CrossRef Medline

Liang X, Zou Q, He Y, Yang Y (2016) Topologically reorganized connectivity architecture of default-mode, executive-control, and salience networks across working memory task loads. Cereb Cortex 26:1501-1511. CrossRef Medline

Meunier D, Lambiotte R, Bullmore ET (2010) Modular and hierarchically modular organization of brain networks. Front Neurosci 4:200. CrossRef Medline

Meunier D, Fonlupt P, Saive AL, Plailly J, Ravel N, Royet JP (2014) Modular structure of functional networks in olfactory memory. Neuroimage 95: 264-275. CrossRef Medline

Newman ME (2006) Modularity and community structure in networks. Proc Natl Acad Sci U S A 103:8577-8582. CrossRef Medline

Nyhus E, Badre D (2015) Memory retrieval and the functional organization of frontal cortex. In: The Wiley handbook on the cognitive neuroscience of memory, 1st edition (Addis DR, Barense M, Duarte A, eds), pp 131149. Hoboken, NJ: Wiley.

Power JD, Cohen AL, Nelson SM, Wig GS, Barnes KA, Church JA, Vogel AC, Laumann TO, Miezin FM, Schlaggar BL, Petersen SE (2011) Functional network organization of the human brain. Neuron 72:665-678. CrossRef Medline

Raichle ME (2015) The brain's default mode network. Annu Rev Neurosci 38:433-447. CrossRef Medline

Rissman J, Wagner AD (2012) Distributed representations in memory: insights from functional brain imaging. Annu Rev Psychol 63:101-128. CrossRef Medline

Rubinov M, Sporns O (2010) Complex network measures of brain connectivity: uses and interpretations. Neuroimage 52:1059-1069. CrossRef Medline

Rugg MD, Vilberg KL (2013) Brain networks underlying episodic memory retrieval. Curr Opin Neurobiol 23:255-260. CrossRef Medline

Schedlbauer AM, Copara MS, Watrous AJ, Ekstrom AD (2014) Multiple interacting brain areas underlie successful spatiotemporal memory retrieval in humans. Sci Rep 4:6431. CrossRef Medline

Smallwood J, Brown K, Baird B, Schooler JW (2012) Cooperation between the default mode network and the frontal-parietal network in the production of an internal train of thought. Brain Res 1428:60-70. CrossRef Medline

Sporns O, Betzel RF (2016) Modular Brain Networks. Annu Rev Psychol 67:613-640. CrossRef Medline 
Spreng RN, Stevens WD, Chamberlain JP, Gilmore AW, Schacter DL (2010) Default network activity, coupled with the frontoparietal control network, supports goal-directed cognition. Neuroimage 53:303-317. CrossRef Medline

Spreng RN, Sepulcre J, Turner GR, Stevens WD, Schacter DL (2013) Intrinsic architecture underlying the relations among the default, dorsal attention, and frontoparietal control networks of the human brain. J Cogn Neurosci 25:74-86. CrossRef Medline

Stanley ML, Dagenbach D, Lyday RG, Burdette JH, Laurienti PJ (2014) Changes in global and regional modularity associated with increasing working memory load. Front Hum Neurosci 8:954. CrossRef Medline

Stevens AA, Tappon SC, Garg A, Fair DA (2012) Functional brain network modularity captures inter- and intra-individual variation in working memory capacity. PLoS One 7:e30468. CrossRef Medline

Vatansever D, Menon DK, Manktelow AE, Sahakian BJ, Stamatakis EA
(2015) Default mode dynamics for global functional integration. J Neurosci 35:15254-15262. CrossRef Medline

Vincent JL, Snyder AZ, Fox MD, Shannon BJ, Andrews JR, Raichle ME, Buckner RL (2006) Coherent spontaneous activity identifies a hippocampal-parietal memory network. J Neurophysiol 96:3517-3531. CrossRef Medline

Vincent JL, Kahn I, Snyder AZ, Raichle ME, Buckner RL (2008) Evidence for a frontoparietal control system revealed by intrinsic functional connectivity. J Neurophysiol 100:3328-3342. CrossRef Medline

Weissman DH, Roberts KC, Visscher KM, Woldorff MG (2006) The neural bases of momentary lapses in attention. Nat Neurosci 9:971-978. CrossRef Medline

Westphal AJ, Reggente N, Ito KL, Rissman J (2016) Unraveling the contributions of rostral prefrontal cortex to analogical reasoning and episodic memory retrieval. Hum Brain Mapp 37:896-912. CrossRef Medline 